\title{
Comportamento do Polipropileno em Presença de Monômeros Trifuncionais no Estado Fundido e sua Influência na Morfologia
}

\author{
Harumi Otaguro, Beatriz W. H. Artel, Duclerc F. Parra, Elizabeth C. L. Cardoso, Luis F. C. P. Lima, Ademar B. Lugão \\ IPEN/CNEN,SP
}

\begin{abstract}
Resumo: $\mathrm{O}$ aumento de ramificações e da massa molecular em polímeros essencialmente lineares influencia as propriedades no estado fundido desses polímeros. Este comportamento foi observado pela análise dos dados de resistência do fundido, extensibilidade e do conteúdo de gel em amostras de Polipropileno linear (iPP) modificado. A modificação foi obtida utilizando agentes modificadores (monômeros multifuncionais) e radiação gama. Os agentes modificadores empregados foram o Tri-metilol-propano-tri-acrilato (TMPTA) e o Tri-alil-cianurato (TAC). O TMPTA demonstrou ser um agente mais eficiente para a promoção do iPP modificado (reticulado/ramificado), uma vez que o produto final apresentou um valor maior da resistência do fundido do que o material com TAC. No entanto, ambos modificam a morfologia original do iPP constatada pelo deslocamento do pico de fusão para temperaturas menores, pelo aumento da temperatura de cristalização (Tc) e pela presença de múltiplas endotermas
\end{abstract}

Palavras-chave: Polipropileno isotático, monômeros multifuncionais, resistência do fundido, radiação gama, reticulações, ramificações e múltiplas endotermas.

\section{The Behavior in the Melt State of Polypropylene (PP) in the Presence of Trifunctional Monomers and their Influence in PP Morphology.}

Abstrac: It well known that in essentially linear polymers the properties of the melt polymers change with increasing molecular weights and degrees of branching. This behavior was observed from data analysis of the strength of the melt polymer, drawability and gel content. In this work isotactic polypropylene (iPP) in the presence of multifunctional monomers was irradiated with gamma radiation. The monomers used were Trimethylolpropane triacrylate (TMPTA) and Triallylcyanurate (TAC). iPP with TMPTA showed the highest strength in the melt and gel content, followed by TAC. It was demonstrated that TMPTA is a powerful agent to crosslinking and branching iPP. Both monomers modified the original morphology of the iPP, the melting peak shifts toward lower temperature and crystallization temperature increased during cooling after the first run. Double melting peaks were observed in the DSC thermograms, which were caused by a double lamellar thickness.

Keywords: Isotactic polypropylene, multifunctional monomers, melt strength, gamma radiation, crosslinking, branching and multiple endoterma.

\section{Introdução}

O Polipropileno linear de configuração isotática (iPP) é um dos polímeros que pode ser objeto de cisão de cadeias durante os processos físicos e químicos utilizados na produção e para a aplicação final. A quebra das macromoléculas de um polímero, usualmente conhecido como "visbreaking"[1] resulta em produtos de baixa massa molecular. Há vários processos que promovem este efeito em poliolefinas, grupo este em que se enquadra o iPP. Dentre estes processos pode-se destacar a pirólise térmi$\mathrm{ca}^{[1]}$, o manuseio de agentes oxidantes durante o processamento $^{[2]}$, o uso da extrusão reativa ${ }^{[2]}$, os múltiplos processamentos ${ }^{[3]}$ como, por exemplo, as extrusões suces- sivas e a utilização da radiação ionizante ${ }^{[2,4-7]}$, utilizados freqüentemente para modificar estruturalmente polímeros que são essencialmente lineares. $\mathrm{O}$ aumento da massa molecular ponderal média pode ocorrer pela reação de recombinação dos radicais ou oligômeros formados durante o processamento. A redução ou ampliação da massa molecular resulta em mudanças nas propriedades físicas e de processamento destes materiais.

O iPP, como já comentado, é um polímero essencialmente linear devido ao seu processo de polimerização que pode utilizar catalisadores do tipo Ziegler-Natta e metalocênicos. Estes processos ainda não permitem a produção do iPP ramificado. No momento, a obtenção de iPP ramificado só é possível via modificação radicalar de suas

Autor para correspondência: Ademar B. Lugão, Laboratório de Polímeros do Centro de Química e Meio Ambiente, IPEN/CNEN, Caixa Postal 11049, CEP: 05422-970, São Paulo, SP. E-mail: ablugao@ipen.br. 
macromoléculas. A presença do polipropileno ramificado no mercado se deve às tecnologias existentes desenvolvidas pela Montell, Borealis (Daploy), Dow Química (Inspire PP) e pelo Ipen/OPP/Embrarad. Os primeiros a iniciarem o desenvolvimento destas tecnologias foram a Montell e a Borealis, utilizando o processo de irradiação com feixe de elétrons e o uso de monômeros ${ }^{[2]}$. Estes produtos constituem uma nova família de polímeros de alto desempenho.

A linearidade das cadeias do iPP confere a este, baixa resistência do fundido (geralmente medido pela força tênsil) o que significa uma baixa resistência ao estiramento durante o processo de elongação. Esta característica limita o uso do iPP em processos que demandam um alto estiramento como, por exemplo, na produção de filmes soprados, produção de espumas, extrusão de revestimentos, produção de peças livres de tensões residuais entre outros. O enxerto de longas ramificações neste polímero confere melhorias na sua viscosidade extensional, no estado fundido ${ }^{[2]}$, resultando no Polipropileno com alta resistência do fundido (HMS-PP). O aumento da resistência do fundido e da extensibilidade do polímero fundido, se deve ao aumento da densidade de emaranhamento das macromoléculas. Este emaranhamento pode ser resultado da presença e da quantidade de ramificações na cadeia, da polidispersividade do material e da reticulação entre cadeias. No entanto, nem sempre o aumento da resistência do fundido vem acompanhado do aumento da extensibilidade como será demonstrado no presente trabalho.

$\mathrm{O}$ processo de modificação do iPP utilizando a radiação ionizante do tipo radiação gama ou feixe de elétrons, pela formação de radicais reativos, no estado sólido é, geralmente, realizado em presença de agentes modificadores ${ }^{[2,6-7]}$ como, por exemplo, os monômeros multifuncionais e/ou agentes oxidantes. Estes agentes modificadores auxiliam no processo de recombinação dos radicais formados durante a irradiação, aumentando com isso a probabilidade de enxertia destes agentes na cadeia principal do polímero e/ou entrecruzamento das cadeias dependendo da concentração do agente utilizado na reação. Geralmente a formação de material reticulado durante a reação pode ser avaliada pela porcentagem de gel formado no sistema em solução. A escolha adequada dos agentes modificadores em paralelo ao controle adequado dos parâmetros de processo será responsável pelo sucesso do produto desejado. Alguns parâmetros que devem ser levados em conta para a seleção destes agentes seriam, por exemplo, suas propriedades de sorção e difusão no sistema a ser empregado.

O presente trabalho propõe a utilização de dois monômeros trifuncionais: o Tri-metilol-propano-triacrilato (TMPTA) e o Tri-alil-cianurato (TAC) como agentes modificadores e controladores da formação de ramificações (ou enxertia) no iPP. A estrutura molecular destes monômeros acrílicos pode ser visualizada na Figura 1. Estes monômeros serão adicionados ao polímero seguido do processo de irradiação ionizante (radiação gama) para a formação de radicais com posterior recombinação dos mesmos para a adição de ramificações (ou enxertia). A eficiência da metodologia proposta será avalia-<smiles>C=CC(=O)OCC(CC)(COC(=O)C=C)COC(=O)C=C</smiles>

(a)<smiles>C=CCOc1nc(OC=C)nc(OC=C)n1</smiles>

(b)

Figura 1. Estrutura molecular dos monômeros acrílicos: (a) TMPTA e (b) TAC.

da em termos do comportamento reológico no estado fundido e do estudo da morfologia destas misturas em comparação ao iPP original.

\section{Experimental}

\section{Material}

Neste trabalho utilizou-se o iPP H603 na forma de peletes (grânulos) produzido e fornecido pela empresa Braskem S/A, com um índice de fluidez de 1,5 g/10 min determinado conforme norma ASTM D1238 $\left(230^{\circ} \mathrm{C}\right.$ e $\left.2,16 \mathrm{~kg}\right)$. Massa molecular ponderal média em torno de $380.000 \mathrm{~g} \cdot \mathrm{mol}^{-1}$.

Os monômeros trifuncionais líquidos estudados neste trabalho foram: o Tri-metil-propano-tri-acrilato (TMPTA) e o Tri-alil-cianurato (TAC) das empresas Cytec e Sartomer na concentração de $1,5 \mathrm{mmol} / 100 \mathrm{~g}$ do polímero. Os monômeros foram utilizados nos ensaios de radiação (fonte de $\mathrm{Co}_{60}$ ) sem prévia purificação.

\section{Preparação das Misturas}

O iPP na forma de grânulos foi misturado aos respectivos monômeros trifuncionais em um misturador rotativo e mantido nestas condições por $24 \mathrm{~h}$. Transcorrido este período a mistura foi processada em uma extrusora (Haake) dupla rosca com o objetivo de homogeneizar o sistema. As temperaturas utilizadas nas 5 zonas foram, respectivamente, 180, 190, 200, 210 e $210^{\circ} \mathrm{C}$

Finalizada a extrusão o material foi transformado em peletes. Na etapa seguinte o material foi irradiado no canal experimental da fonte de $\mathrm{Co}_{60}$ da empresa Embrarad, atualmente com $700.000 \mathrm{Ci}$. A taxa de dose foi de $5 \mathrm{kGy}$. $\mathrm{h}^{-1}$, sendo irradiados à temperatura ambiente. A dose total utilizada foi de 10 e $20 \mathrm{kGy}$ medida com dosimetro Red Perspex 4034 da Harwell. Após o processo de irradiação as amostras foram novamente processadas na extrusora, e, finalmente, disponíveis para os estudos reológicos e morfológicos. 


\section{Determinação da Porcentagem de Gel}

Foram pesados em torno de $0,5 \mathrm{~g}$ da mistura de iPP com os respectivos agentes TMPTA e TAC para a determinação da porcentagem de gel. Os materiais pesados foram envoltos em poliéster formando pequenos pacotes, e acondicionados em um balão contendo xileno. O sistema foi mantido em refluxo durante $24 \mathrm{~h}$ à temperatura de ebulição do solvente. Finalizado o período de $24 \mathrm{~h}$ o polímero foi retirado do balão e mantido por $12 \mathrm{~h}$ em um dessecador para posterior pesagem e determinação da fração gel.

\section{Determinação da Resistência do Fundido.}

A resistência do fundido (RF) e a extensibilidade destas misturas foram obtidas em um reômetro Rheotens da Göttfert alocado no laboratório da empresa Braskem. As condições experimentais utilizadas para a determinação da resistência do fundido foram as seguintes: temperatura de extrusão de $190^{\circ} \mathrm{C}$; velocidade de extrusão de 5,0 rpm; diâmetro do capilar de $2,90 \mathrm{~mm}$; comprimento do capilar de $18,09 \mathrm{~mm}$ e a distância entre matriz e as engrenagens de $6,97 \mathrm{~cm}$. Foram obtidos os resultados de força tênsil $(\mathrm{cN})$ e extensibilidade $(\mathrm{cm} / \mathrm{s})$.

\section{Análise do Comportamento de Fusão}

Foi utilizado um equipamento de DSC marca METTLER TOLEDO MOD. $822^{\mathrm{e}}$ para o estudo do comportamento de fusão e da morfologia destas amostras. Neste estudo foram analisadas amostras com cerca de $20 \mathrm{mg}$, utilizando uma taxa de aquecimento de $10{ }^{\circ} \mathrm{C} / \mathrm{min}$, partindo da temperatura de -20 a $200{ }^{\circ} \mathrm{C}$ sob atmosfera de nitrogênio $\left(50 \mathrm{~mL} \mathrm{~min}{ }^{-1}\right)$. À temperatura de $200{ }^{\circ} \mathrm{C}$ foi realizada uma isoterma de 2 min para eliminação da memória térmica das amostras (proveniente da polimerização ou do processamento, a presença de agentes nucleantes e a existência da transcristalinidade). Em seguida as amostras foram resfriadas a $-50{ }^{\circ} \mathrm{C} / \mathrm{min}$ até à temperatura de $-20^{\circ} \mathrm{C}$. Atingida esta temperatura iniciou-se a segunda varredura nas mesmas condições da primeira a fim de analisar os seguintes parâmetros: índice de cristalinidade $\left(\mathrm{X}_{\mathrm{c}}\right)$, valores médio da temperatura de fusão $\left(\mathrm{T}_{\mathrm{f}}\right)$, da temperatura de cristalização $\left(T_{c}\right)$ e da entalpia envolvida no processo de fusão $\left(\Delta H_{f}\right)$. Neste estudo foi utilizado para o cálculo do índice de cristalinidade das misturas o valor de $\Delta \mathrm{H}$ de $190 \mathrm{~J} / \mathrm{g}^{[8]}$ para o iPP $100 \%$ cristalino. O equipamento foi devidamente calibrado utilizando padrão de Índio ${ }^{[9]}\left(\mathrm{T}_{\mathrm{f}}=156,61{ }^{\circ} \mathrm{C}\right.$; $\left.\Delta \mathrm{H}=28,54 \mathrm{~J} \mathrm{~g}^{-1}\right)$ sob atmosfera de nitrogênio.

\section{Resultados e Discussões}

A presença de ramificações (enxertia) e/ou reticulações formadas durante o processo de irradiação (com radiação ionizante de fonte gama) para o iPP em presença de monômeros acrílicos pode ser avaliada, em primeira instância, pelo conteúdo de gel formado nestas misturas, como verificado nos trabalhos de Rätzsch ${ }^{[2]}$, Chapiro ${ }^{[4]}$ e Lyons ${ }^{[5]}$. Os resultados obtidos para as misturas irradiadas a distintas doses de radiação estão apresentados na Tabela 1.
Tabela 1. Conteúdo de gel das amostras de iPP irradiadas sob atmosfera de ar à temperatura ambiente em presença dos monômeros líquidos TAC e TMPTA

\begin{tabular}{lccc}
\hline Amostras & $\begin{array}{c}\text { Dose de radiação } \\
(\mathbf{k G y})\end{array}$ & $\begin{array}{c}\text { Porcentagem de gel } \\
(\boldsymbol{\%})\end{array}$ & $\begin{array}{c}\text { Erro } \\
( \pm)\end{array}$ \\
\hline iPP Puro & - & - & - \\
iPP + TAC & 10 & 13,0 & 10,0 \\
iPP + TAC & 20 & 17,0 & 2,5 \\
iPP + TMPTA & 10 & 40,0 & 7,0 \\
iPP + TMPTA & 20 & 25,0 & 2,0 \\
\hline
\end{tabular}

Estes monômeros acrílicos (TMPTA e TAC) são eficientes agentes promotores de reticulação e enxertia em iPP, o que foi confirmado pelos resultados do conteúdo de gel destas amostras. O TMPTA apresentou uma maior reatividade com o polímero do que o TAC para as duas doses estudadas, evidenciadas pelo maior valor da porcentagem de gel. Isto, provavelmente, está relacionado com a estrutura molecular do TMPTA, que parece ser semelhante à do iPP. Esta similaridade (vide Figura 1) facilita a reatividade entre os dois reagentes quando irradiados com radiação ionizante do tipo gama. Com relação ao monômero TAC a menor reatividade pode ser explicada em termo estrutural, pela perda através da volatilidade ou formação de oligômeros.

No que diz respeito às doses estudadas, $10 \mathrm{kGy}$ foi a que apresentou maior porcentagem de gel para os monômeros estudados. Para o TMPTA a dose de 20 kGy parece ser a dose limite, pois, a partir deste valor, parece haver uma maior predominância do fenômeno de degradação do polímero do que o de recombinação pela ramificação ou reticulação. No caso do TAC, também se esperava um valor maior da porcentagem de gel para as duas doses utilizadas, próximo ao valor do TMPTA, visto que os dois monômeros são trifuncionais, e, segundo a literatura $^{[2,6]}$, estes são os que apresentam maior reatividade em comparação aos mono e bifuncionais. Porém, o resultado obtido para o TAC foi inferior ao do TMPTA, ressaltando a diferença de reatividade entre os dois monômeros multifuncionais para a modificação do iPP. Os monômeros acrílicos selecionados neste trabalho evidenciaram uma modificação no iPP como bem visto pelos valores da porcentagem de gel, esta alteração irá influenciar no comportamento do fluxo destes materiais. Esta propriedade foi avaliada pelo resultado da resistência do fundido e da extensibilidade.

A resistência do fundido caracteriza o comportamento de um polímero no estado fundido sob tensão, e a força obtida no ponto de ruptura deste material é um indicativo do desempenho extensional relativo nesta condição ${ }^{[2,10]}$. Se o TMPTA é o monômero mais reativo para o iPP nas condições estudadas é de se esperar que apresente o maior valor de resistência do fundido. Este comportamento pode ser avaliado pelos dados que se encontram dispostos na Tabela 2.

A mistura de iPP irradiado a $10 \mathrm{kGy}$ em presença do agente TMPTA apresentou um aumento na resistência do fundido em torno de quatro vezes o valor do material original (puro). Seguido em menor escala para a dose de $20 \mathrm{kGy}$ para os dois 
Tabela 2. Dados de resistência do fundido e extensibilidade obtidos a $190{ }^{\circ} \mathrm{C}$ para os Polipropilenos modificados.

\begin{tabular}{lccc}
\hline Amostras & $\begin{array}{c}\text { Dose de radiação } \\
(\mathbf{k G y})\end{array}$ & $\begin{array}{c}\text { Força Tênsil } \\
(\mathbf{c N})\end{array}$ & $\begin{array}{c}\text { Extensibilidade } \\
(\mathbf{c m} / \mathbf{s})\end{array}$ \\
\hline iPP Puro & - & 16,3 & 10,3 \\
iPP + TAC & 10 & 31,2 & 7,2 \\
iPP + TAC & 20 & 49,9 & 11,6 \\
iPP + TMPTA & 10 & 62,8 & 9,1 \\
iPP + TMPTA & 20 & 48,4 & 10,4 \\
\hline
\end{tabular}

monômeros e, finalmente, a amostra de TAC irradiada a 10 $\mathrm{kGy}$, que apresentou menor valor. iPP mais TMPTA (10 kGy) $>$ iPP mais TMPTA (20 kGy), iPP mais TAC (20 kGy) > iPP mais TAC (10 kGy). Os dados de resistência do fundido apresentado na Tabela 2 vêm corroborar com os dados da porcentagem de gel apresentados na Tabela 1. No entanto, os dados de extensibilidade para estes acrilatos estão próximos. A viscosidade extensional ${ }^{[10]}$ pode ser entendida como uma medida da resistência do fundido ao ser estirado. Segundo Lugão et al. ${ }^{[7]}$ a extensibilidade do iPP puro obtida a $190{ }^{\circ} \mathrm{C}$ é da ordem de $10,3 \mathrm{~cm} \mathrm{~s}^{-1}$. Neste sentido a amostra que apresentou maior resistência do fundido (TMPTA 10 kGy) não apresentou uma grande variação em sua extensibilidade em comparação ao material puro. No caso da amostra de TAC 10 kGy sua extensibilidade foi menor que o material original. Para estas duas condições o aumento da força tênsil destas amostras provavelmente seja resultado do alto índice de reticulação (aumento da porcentagem de gel). No caso do TAC 20 kGy e TMPTA $20 \mathrm{kGy}$, apresentaram valores de extensibilidade iguais ao do polímero puro. Porém, o aumento na força tênsil provavelmente seja a combinação do grau de ramificação e do efeito de reticulação resultante do processo.

Com relação a extensibilidade a amostra de $10 \mathrm{kGy}$, tanto para o TAC como o TMPTA, perde extensibilidade apesar de reticular como mostra o conteúdo de gel, sugerindo pouca influência na formação de ramificação. Já no caso das amostras de $20 \mathrm{kGy}$ pode-se dizer que a reticulação combinada à ramificação estejam influenciado na extensibilidade. A determinação das propriedades reológicas destas amostras em regime oscilatório e transiente constituirá o escopo de um estudo futuro.

O comportamento de fusão destas amostras tanto na primeira quanto na segunda varredura sem a realização de um método de cristalização, quer seja isotérmica ou nãoisotérmica, é praticamente o mesmo. A cristalização que ocorre nestas amostras é aquela observada durante o segmento de resfriamento no programa utilizado no DSC. Na Figura 2 pode ser visualizado o intervalo da segunda varredura das curvas de DSC das amostras.

Observa-se um deslocamento da temperatura máxima do pico de fusão de todas as amostras de HMS-PP, para temperaturas menores (vide Figura 2 e resultados na Tabela 3). Isto é um indicativo de que os cristais, formados durante o resfriamento destas amostras nos ensaios de DSC, são menores e defeituosos. Provavelmente a presença de ramificações e de entrecruzamento (reticulações) na cadeia principal do polímero influencia na formação destes cristais, dificultando a dobra da cadeia e a reentrância adjacente na superfície em crescimento do cristal lamelar (na direção de um dos eixos cristalográficos, eixo b). O material puro em geral apresenta um único pico de fusão em uma curva de DSC sem qualquer tipo de cristalização, como observado na curva (a) da Figura 2. As curvas referentes aos distintos HMS-PP se assemelham ao material puro com exceção da amostra de TMPTA irradiado a $20 \mathrm{kGy}$, que apresenta um ombro mais acentuado a mais baixa temperatura, assinalado pela seta na curva (e). Nesta curva a sobreposição dos picos (múltipla endoterma) é mais acentuada que nas demais curvas.

A presença das múltiplas endotermas ${ }^{[11]}$ tem recebido diferentes interpretações com relação à sua origem e, principalmente, no que diz respeito ao pico que apresenta a menor temperatura de fusão. Estas interpretações, geralmente, são reunidas em grupos e descritas por meio de modelos: (i) da existência de diferentes estruturas cristalinas ${ }^{[12]}$, (ii) de fusão e recristalização ${ }^{[11,13-15]}$ e (iii) da dupla população de espessuras lamelares ${ }^{[13,16]}$, além do modelo de envelhecimento físico ${ }^{[14]}$, de diferentes morfologias ${ }^{[11]}$ e do modelo de relaxação da fase "pseudo cristalina"[17]. Todos estes modelos estão fundamentalmente relacionados ao aparecimento, em muitos polímeros, de dois picos endotérmicos nas curvas de DSC. Em geral, a validade destes modelos deve ser considerada para cada caso em particular ${ }^{[18]}$. No caso do presente trabalho o modelo que melhor se aplica à presença das endotermas é o modelo da dupla população de espessuras lamelares. Esta diferença de população se deve, em parte, à redução da massa molecular do polímero no processo de irradiação, a qual aumenta com a dose utilizada. Estas cadeias como apresentam uma massa molecular menor cristaliza a mais alta temperatura no resfriamento, formando cristais de tamanhos inferiores e imperfeitos. A presença deste pico (endoterma a mais baixa temperatura) nas condições analisadas no presente trabalho está de acordo com o processo de foto-degradação observado no iPP quando exposto à radiação ultra-violeta (UV) por longos períodos descrito nos

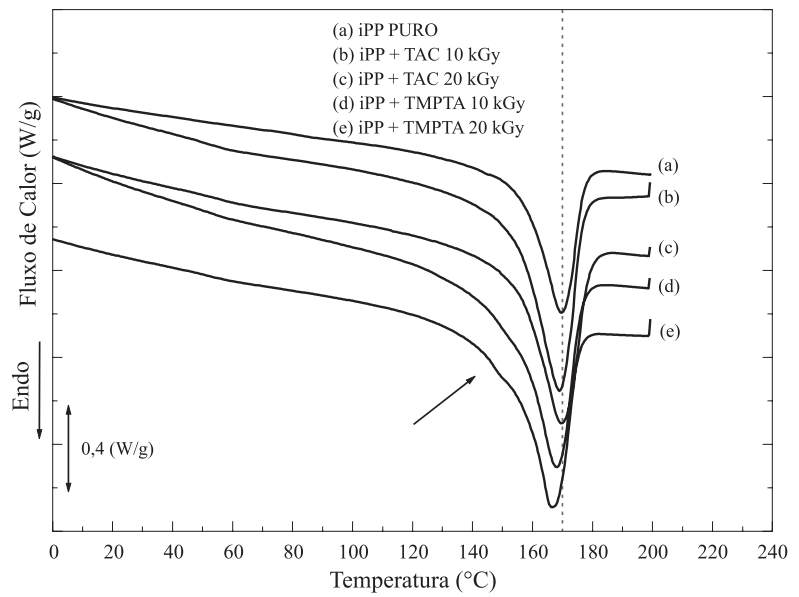

Figura 2. Curvas de DSC das distintas misturas e do iPP puro obtidas na segunda varredura. 
trabalhos de Rabello e White ${ }^{[19,20]}$. Com o tempo de exposição à radiação UV há um aumento do processo degradativo (quebra das cadeias) facilitando a presença de defeitos químicos que podem ser introduzidos na cadeia do polímero. Como a intensidade da radiação (radiação gama) utilizada neste trabalho é superior à radiação UV é de se esperar um aumento no processo de degradação e a presença de defeitos na cadeia do polímero como um todo.

A presença destes defeitos, de entrecruzamento e prováveis ramificações influenciam não só na presença das múltiplas endotermas como nas propriedades térmicas do HMS-PP. Este efeito pode ser avaliado pelos resultados dos parâmetros apresentados na Tabela 3. O índice de cristalinidade das distintas amostras foi calculado utilizando a seguinte expressão:

$$
X_{c}=\left(\frac{\Delta H_{f}}{\Delta H_{f}^{0}}\right) \times 100
$$

onde o $\Delta H_{f}^{0}$ corresponde ao valor de entalpia de fusão para o iPP $100 \%$ cristalino $(190 \mathrm{~J} / \mathrm{g})^{8}$. O valor do $\Delta \mathrm{H}_{\mathrm{f}}$ de cada amostra foi obtido traçando a linha de base partindo da região da curva logo após a $\mathrm{T}_{\mathrm{g}}$ (região em que não há desvio da linha de base), estendendo-se até o término do pico de fusão, conforme método proposto por Khanna \& $\mathrm{Kuhn}^{[21]}$.

Todas as amostras analisadas apresentaram valores de entalpia de fusão e, conseqüentemente, do índice de cristalinidades superiores ao polímero puro, o que era de se esperar dado à existência do processo de degradação. Outro fator que pode estar contribuindo para o aumento da cristalinidade é a presença dos monômeros remanescentes no meio reacional que podem estar atuando como agentes de nucleação. A presença do agente nucleante na massa polimérica desloca a temperatura máxima do pico de cristalização para valores superiores ao polímero original (Tabela 3) durante o processo de resfriamento, facilitando a cristalização. Por outro lado, para os resultados apresentados neste trabalho a presença de entrecruzamentos e ramificações no iPP também estão atuando como agente de nucleação. Esta evidência é comprovada pelo deslocamento da $T_{c}$ para temperaturas superiores à do polímero original, resultado este que está de acordo com o trabalho de Rätzsch ${ }^{[2]}$. O autor deste trabalho demonstrou que o HMS-PP atua como um

Tabela 3. Dados das propriedades térmicas dos HMS-PP obtidos utilizando-se a técnica de calorimetria exploratória diferencial a $10^{\circ} \mathrm{C} / \mathrm{min}$

\begin{tabular}{lccccc}
\hline Misturas & $\begin{array}{c}\text { Dose de radiação } \\
(\mathbf{k G y})\end{array}$ & $\begin{array}{c}\mathbf{T}_{\mathbf{f}} \\
\left({ }^{\circ} \mathbf{C}\right)\end{array}$ & $\begin{array}{c}\mathbf{T}_{\mathrm{c}} \\
\left({ }^{\circ} \mathbf{C}\right)\end{array}$ & $\begin{array}{c}\Delta \mathbf{H}_{\mathrm{f}} \\
(\mathbf{J} / \mathbf{g})\end{array}$ & $\begin{array}{c}\mathbf{X}_{\mathrm{c}} \\
(\boldsymbol{\%})\end{array}$ \\
\hline iPP Puro & - & 172,2 & 95,4 & 62,0 & 32,6 \\
iPP + TAC & 10 & 170,3 & 111,9 & 64,4 & 33,9 \\
iPP + TAC & 20 & 169,1 & 109,5 & 66,3 & 34,8 \\
iPP + TMPTA & 10 & 171,5 & 109,5 & 64,9 & 34,1 \\
iPP + TMPTA & 20 & 169,0 & 110,0 & 68,4 & 36,0 \\
\hline
\end{tabular}

agente nucleante quando misturado ao homopolímero de iPP. Porém, como anteriormente comentado os entrecruzamentos e as ramificações nas cadeias são os responsáveis pela mudança no comportamento do fundido (resistência do fundido) das amostras.

\section{Conclusões}

Os monômeros de acrilatos em especial o TMPTA e o TAC são eficientes agentes promotores de reticulação e provavelmente de enxertia em iPP nas doses de 10 e 20 kGy, analisados através da porcentagem de gel, de sua extensibilidade e da força tênsil destas amostras.

A presença de ramificações no iPP linear pode ser inferida pelo aumento da resistência do fundido mais extensibilidade (20 kGy) destas amostras em comparação ao material puro. Este mesmo comportamento é observado nos valores da porcentagem de gel obtidos para os HMS-PP.

Estes monômeros multifuncionais afetam a morfologia do iPP o que pode ser constatado pelo deslocamento do pico de fusão para temperaturas menores e pela presença das múltiplas endotermas. A endoterma que funde a mais baixa temperatura, é nítida na amostra contendo TMPTA, e pode ser interpretada como sendo resultado da dupla população de espessuras lamelares. Isto se deve em grande parte à redução da massa molecular do polímero ocorrida durante o processo de radiação e pela presença de moléculas defeituosas. Outra característica observada foi o deslocamento da $T_{c}$ para temperaturas superiores ao polímero puro (durante o resfriamento) demonstrando o efeito de nucleação dos monômeros remanescentes, ramificações e de reticulações presentes.

\section{Agradecimentos}

Os autores agradecem a Braskem S/A pelo iPP fornecido e pela realização dos ensaios de resistência do fundido e extensibilidade das misturas.

\section{Referências Bibliográficas}

1. Willians, S. D. - U. S. Patent. 1998, 5.820.981.

2. Rätzsch, M. et al. - Prog. Polym. Sci., 27, p.1195 (2002).

3. Canevarolo, S. V. - Polym. Degrad. Stab., 709, p.71 (2000).

4. Chapiro, A. - Radiation Chemistry of Polymeric Systems. Interscience, New York (1962).

5. Lyons, B. J. - J. Polym. Sci. Part A., 3, p.777 (1965).

6. Yoshii, F. et al. - J. Appl. Polym. Sci., 60, p.617 (1996).

7. Lugão, A. B. et al. - Radiat. Phys. and Chem., 63, p.509 (2002).

8. Amash, A. \& Zugenmaier, P. - J. Appl. Polym. Sci., 63, p.1143 (1997). 
9. Haines, P. J. - Thermal Methods of Analysis - Principle, applications and problems. Blackie Academic \& Professional, New York (1995).

10. Muke, S., Ivanov, I. , Kao, N. \& Bhattacharya, S. N. - J. Non-Newtonian Mech., 101, p. 77 (2001).

11. Verma, R. K. \& Hsiao, B. S. - Trip., 4, p.312 (1996).

12. Bell, J. P. \& Murayama, T. - J. Polym. Sci. Part A(2)., 7, p.1059 (1969).

13. Bassett, D. C. et al. - Polymer., 29, p.1745 (1988).

14. Velikov, V. \& Marand, H. - J. Thermal Analysis., 49, p.375 (1997).

15. Sohn, S., Alizadeh, A. \& Marand, H. - Polymer., 41, p.8879 (2000).

16. Cebe, P. \& Hong, F. D. - Polymer., 27, p.1183 (1986).
17. Bonnet, M. Rogausch, K. D. \& Petermann, J. - Colloid Polym. Sci., 277, p.513 (1999).

18. Otaguro, H. - "Alguns problemas relacionados à cristalinidade em polímeros e suas correlações com o processamento e a morfologia: $\mathrm{O}$ caso do Poliestireno Isotático", Tese de doutorado, Universidade Federal de São Carlos, Brasil (2002).

19. Rabello, M. S. \& White, J. R. - Polymer., 38, p.6379 (1997).

20. Rabello, M. S. \& White, J. R. - Polymer., 38, p.6389 (1997).

21. Khanna, Y. P. \& Kuhn, W. P. - J. Polym. Sci: Polym. Phys., 35, p.2219 (1997).

Enviado: 24/11/03

Aprovado: 31/03/04 